\title{
Courants induits et dissipation de l'énergie des vagues sur les plages macrotidales de la mer d'Iroise
}

\author{
Aurélie Dehouck* - Hélène Dupuis** - Nadia Sénéchal** \\ * Géomer, UMR6554 LETG, Université de Bretagne Occidentale, Institut \\ Universitaire Européen de la Mer, Technopôle Brest Iroise, 29280 Plouzané \\ aureliedehouck@voila.fr \\ ** Methys, UMR5805 EPOC, Université Bordeaux 1, avenue des facultés, 33405 \\ Talence cedex \\ h.dupuis@epoc.u-bordeaux1.fr \\ n.senechal@epoc.u-bordeauxl.fr
}

\begin{abstract}
RÉSUMÉ. Plusieurs campagnes de mesures morphologiques et hydrodynamiques se sont déroulées aux printemps 2004 et 2005 sur quatre plages de la mer d'Iroise (Finistère). A partir de ces quatre jeux de données (élévation de la surface libre, vitesse du courant crossshore et longshore), l'évolution de la dissipation de l'énergie des vagues dans le temps (au cours du cycle de marée) et l'espace (à travers la zone intertidale) est étudiée. Ces résultats sont comparés aux paramétrisations empiriques de la hauteur relative des vagues $\gamma_{s}$ (Raubenheimer et al., 1996; Sénéchal et al., 2001), paramètre utilisé dans les modèles hydrodynamiques. Enfin, le critère de déferlement local $\gamma_{s, b}$ permet de caractériser les processus hydrodynamiques (asymétrie des vagues, courant de retour induit par le déferlement) impliqués dans le transport sédimentaire en zone intertidale.

ABSTRACT. Several field experiments of both morphological and hydrodynamical measurements occured during springs 2004 and 2005 on four beaches of the Iroise Sea (Finistère). Within these four datasets (sea surface elevation, cross-shore and longshore flow velocities), dissipation of wave energy is investigated through time (accounting with tidal cycle) and space (across the intertidal zone). These results are compared to empirical parametrizations of relative wave height $\gamma_{s}$ (Raubenheimer et al., 1996 ; Sénéchal et al., 2001), parameter greatly used in hydrodynamic models. Then, the local breaking criteria $\gamma_{s, b}$ is used to characterize wave-related processes (wave asymetry, breaking-induced undertow) involved in sediment transport in the intertidal zone.
\end{abstract}

MOTS-CLÉS : courants, vagues, dissipation, régime macrotidal, zone intertidale, Bretagne KEYWORDS: currents, waves, energy dissipation, macrotidal regim, intertidal zone, Brittany

Revue européenne de génie civil. Génie côtier $-\mathrm{n}^{\circ} 1 / 2007$, pages 1 à $\mathrm{X}$ 


\section{Introduction}

Sur les plages macrotidales, la marée module l'élévation du plan d'eau et provoque le déplacement de la zone d'action des processus hydrodynamiques à travers la zone intertidale (Masselink, 1993 ; Masselink et Turner, 1999). A un instant donné, l'hydrodynamisme de la zone intertidale est donc dominé par divers processus qui sont eux-mêmes contrôlés par la dissipation de l'énergie des vagues. Celle-ci est souvent quantifiée par la hauteur relative des vagues $\gamma_{\mathrm{s}}$, définie comme le rapport de leur hauteur significative $\mathrm{H}_{\mathrm{s}}$ à la hauteur d'eau $\mathrm{h}$ (Osborne et Greenwood, 1992; Ruessink et al., 1998). De nombreux travaux s'y sont intéressés dans le but de connaître les paramètres contrôlant l'atténuation de la hauteur des vagues en zone littorale et d'intégrer ces paramétrisations dans les modèles hydrodynamiques. Les premiers d'entre eux considéraient que la hauteur relative des vagues $\gamma_{\mathrm{s}}$ était constante (Thornton et Guza, 1982; 1983) ou diminuait (Andersen et Fredsoe, 1983) lors de la propagation des vagues dans la zone de surf. Par la suite, d'autres travaux ont montré que la hauteur relative des vagues est bien corrélée avec le paramètre $\beta / \mathrm{kh}$, pente locale normalisée par la hauteur d'eau (Raubenheimer et al., 1996; Sénéchal et al., 2001 ; 2005). Ces améliorations successives de l'expression de la dissipation de l'énergie des vagues dans les petits fonds possèdent un intérêt majeur pour les applications en ingénierie côtière dont les modèles hydrodynamiques et de transport sédimentaire sont les principaux outils d'investigation.

Les objectifs de cette étude sont: (1) d'analyser la dissipation de l'énergie des vagues lors de leur propagation sur les plages en comparant nos données aux paramétrisations existantes (Raubenheimer et al., 1996; Sénéchal et al., 2001 ; 2005) ; (2) d'utiliser le paramètre $\gamma_{s}$ pour identifier les processus hydrodynamiques impliqués dans le transport sédimentaire en zone intertidale.

\section{Contexte environnemental}

\subsection{Caractères morphologiques}

La mer d'Iroise est un espace maritime ouvert localisé à la pointe occidentale de la Bretagne (Finistère), délimité au Nord par l'île d'Ouessant, au Sud par le raz de Sein (fig. 1). Son littoral est rocheux, extrêmement découpé du fait de l'érosion différentielle des roches et de la tectonique locale qui ont façonné l'évolution géomorphologique de cette côte. Les plages sableuses «de poche» sont pour la plupart de dimension réduite (quelques dizaines à centaines de mètres d'extension longitudinale). Au cours des fluctuations saisonnières de la morphologie des plages (Dehouck et Rihouey, soumis), sous les sables fins à grossiers $\left(d_{50}=250-600 \mu \mathrm{m}\right)$ peuvent découvrir des cordons de galets, des platiers rocheux ou des niveaux de tourbes. Les plages de Corsen et Tregana sont de type réflectif, la plage de Porsmilin de type intermédiaire (terrasse de basse mer dans la classification de 
Masselink et Short (1993)) et les Blancs Sablons de type dissipatif. On y rencontre des formes d'accumulation sédimentaire, barres de swash ponctuelles à Porsmilin sur les niveaux de basse mer et de haute mer, système simple ou multiple de croissants de plage à Tregana.

\subsection{Caractères hydrodynamiques}

La mer d'Iroise est caractérisée par un régime macrotidal semi-diurne dont les marnages varient entre $3 \mathrm{~m}$ en marées de mortes-eaux (coefficient 45) et $7 \mathrm{~m}$ en vives-eaux exceptionnelles (coefficient 120). Au large de l'île d'Ouessant, les conditions de mer sont très agitées $\left(\mathrm{H}_{\mathrm{s}, 95 \%}=5,9 \mathrm{~m}, \mathrm{H}_{\mathrm{s}, 99 \%}=8,3 \mathrm{~m}\right.$ à partir des données de l'Atlas Numérique de Houle sur la période 1979-2002, source : LNHE) (fig. 1). En effet, les houles générées en Atlantique Nord parviennent sous une incidence ouest tandis que le passage des systèmes dépressionnaires engendre une forte mer $\mathrm{du}$ vent locale. Toutefois, l'agitation modale sur les plages étudiées demeure relativement faible $\left(\mathrm{H}_{\mathrm{s}}<0,5 \mathrm{~m}\right) \mathrm{du}$ fait des nombreux mécanismes affectant la propagation des vagues vers la côte (Dehouck, 2006).

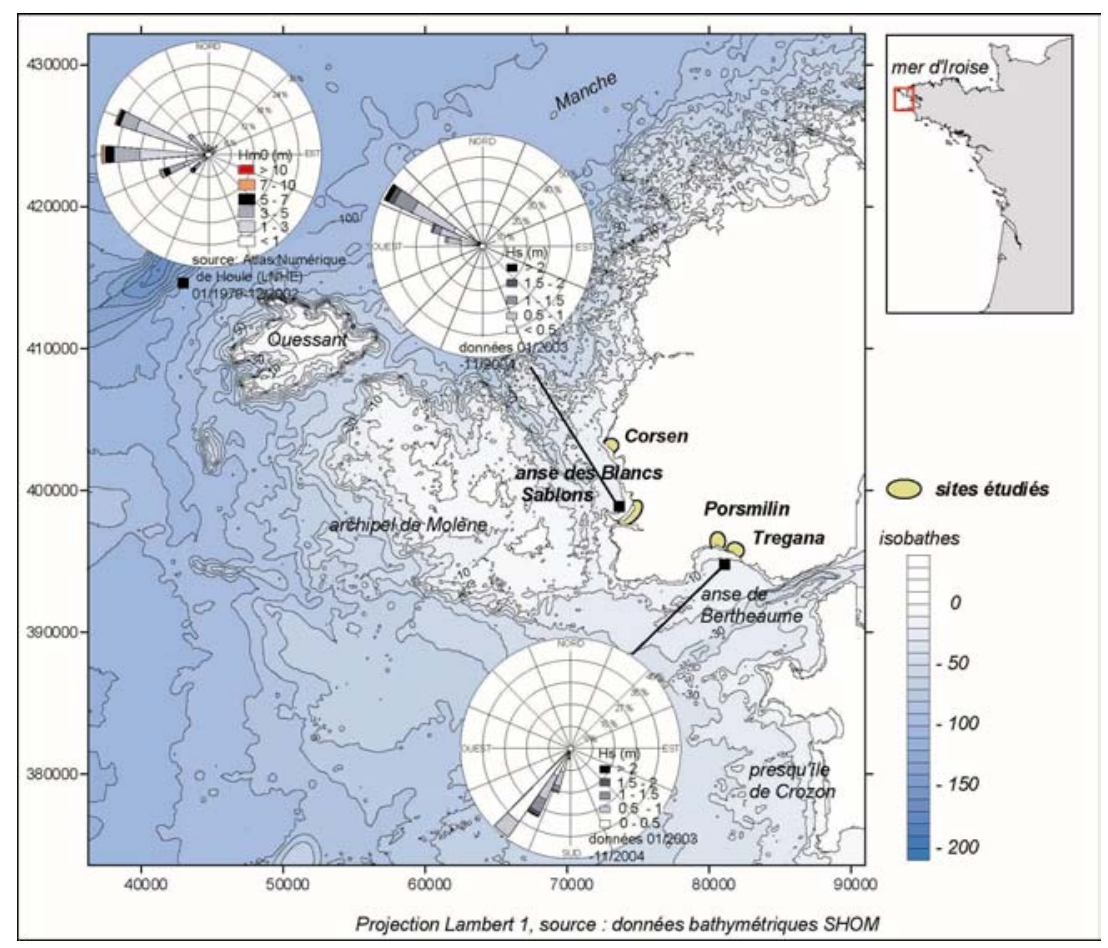

Figure 1. Cadre géographique de la zone d'étude et roses des houles au large de l'île d'Ouessant, dans les anses des Blancs Sablons et de Bertheaume. 


\section{Méthodes d'acquisition et d'analyse des données}

\subsection{Présentation des campagnes de mesure}

Les données analysées dans cette étude sont issues de quatre campagnes de mesure réalisées aux printemps 2004 et 2005 lors de conditions énergétiques variées. L'alternance de coups de vents $\left(\mathrm{H}_{\mathrm{s}}=1\right.$ à $1,5 \mathrm{~m}$ en zone intertidale) et de périodes de calme $\left(\mathrm{H}_{\mathrm{s}}=0,3-0,4 \mathrm{~m}\right)$ a été observée sur les quatre plages. Un capteur S4DW a été déployé sur la basse plage à Porsmilin, Corsen et Tregana et par $-6 \mathrm{~m}$ C.M. (côte marine) aux Blancs Sablons, entre $+0,45 \mathrm{~m}$ à $+0,55 \mathrm{~m}$ au-dessus du fond et pendant 1 à 4 semaines selon les sites. En parallèle, des mesures topographiques ont été réalisées pour étudier l'évolution morphologique de la zone intertidale sur de courtes échelles de temps. Ces résultats sont partiellement exposés par Dehouck et al. (2005) et détaillés par Dehouck (2006).

\subsection{Méthodes d'analyse}

Les données d'élévation de la surface libre, découpées en séries de 10 à 20 min, sont pré-analysées en prenant soin d'éliminer les acquisitions durant lesquelles le capteur était émergé, de les corriger de sa hauteur, de la pression atmosphérique et de filtrer le signal de marée (suppression de la tendance linéaire sur chaque série de 10-20 min). La hauteur significative des vagues $H_{s}$ est calculée par intégration du spectre d'énergie des vagues dans la bande fréquentielle $0,05-0,5 \mathrm{hz}$ correspondant à la houle et à la mer du vent. Les bornes d'intégration du spectre d'énergie des vagues sont définies avec attention car elle conditionnent les écarts entre les différentes paramétrisations de la hauteur relative des vagues (Sénéchal et al., 2005). Le paramètre $\beta / \mathrm{kh}$ est défini par la hauteur d'eau h, la pente de la plage $\beta$ sur une distance équivalant à une longueur d'onde en aval du capteur et le nombre d'onde $\mathrm{k}$ associé à la fréquence centroïdale $\left(\mathrm{f}_{\mathrm{c}}\right)$. Ces deux paramètres se calculent respectivement selon les expressions [1] et [2] où L est la longueur d'onde en eaux peu profondes, g l'accélération de la gravité, $m_{n}$ le moment d'ordre $n$ et $S(f)$ le spectre de densité d'énergie des vagues. Les composantes cross-shore U et longshore $\mathrm{V}$ du courant sont analysées dans le domaine temporel et moyennées sur des séries de 10 ou $20 \mathrm{~min}$.

$$
\begin{aligned}
& k=\frac{2 \pi}{L}=\frac{2 \pi f_{c}}{\sqrt{g h}} \\
& f_{c}=\frac{m_{1}}{m_{0}}=\frac{\int_{0,05}^{0,5} S(f) f d f}{\int_{0,05}^{0,5} S(f) d f}
\end{aligned}
$$




\section{Résultats et discussion}

\subsection{Dissipation de l'énergie des vagues}

La figure 2 indique la hauteur relative des vagues fonction de la hauteur d'eau locale dont les valeurs varient de 0 à 1,2 sur les plages de Porsmilin, Corsen, Tregana et des Blancs Sablons au cours des campagnes de mesure. L'ensemble des données des quatre campagnes de mesures est représenté toutes conditions hydrodynamiques confondues. Aux Blancs Sablons, la hauteur relative des vagues est toujours inférieure à 0,2 ce qui est caractéristique de conditions d'énergie faibles en zone de levée des vagues. Sur les trois autres plages, la hauteur relative des vagues augmente avec des profondeurs d'eau décroissantes malgré l'atténuation simultanée de leur hauteur à travers la zone de déferlement. D'un point de vue qualitatif, ces résultats sont cohérents avec ceux de Raubenheimer et al. (1996) et Sénéchal et al. (2001). Par ailleurs, on constate une variabilité de la forme du nuage de points et de la valeur maximale atteinte entre les trois sites. Ceci traduit certainement une hétérogénéité des conditions d'énergie rencontrées pendant les diverses expériences et des différences d'ordre morphologique (pente) entre les plages.

La figure 3 représente la hauteur relative des vagues fonction de la pente locale normalisée par la hauteur d'eau $(\beta / \mathrm{kh})$. Seules les données acquises en zone de déferlement y figurent. Ce paramètre adimensionnel prend en considération l'influence de la pente de la plage, de la hauteur d'eau locale et des conditions de vagues (via le nombre d'onde $\mathrm{k}$ ) sur la hauteur relative des vagues en zone de déferlement. Les trois jeux de données acquis à Porsmilin, Corsen et Tregana sont assemblés en dix classes de pente locale normalisée $(\beta / \mathrm{kh})$ d'intervalle 0,05 de manière à gommer la grande dispersion des données et à extraire des valeurs moyennes (symbolisées par des cercles pleins sur la fig. 3). En effectuant une régression linéaire sur ces valeurs moyennées, on obtient la relation [3] établissant une corrélation positive entre la hauteur relative des vagues en zone de déferlement et la pente locale normalisée (coefficient de corrélation de 0,76).

$$
\gamma_{s}=0,30+1,06 \beta / k h
$$

Cette nouvelle relation est valable pour des pentes normalisées comprises entre 0 et 0,5. Elle se situe entre les paramétrisations de Raubenheimer et al. (1996) et Sénéchal et al. $(2001,2005)$ dont les expressions sont formulées par les équations [4] et [5] et reportées sur la figure 3 par des droites en pointillés.

$$
\begin{aligned}
& \gamma_{s}=0,19+1,05 \beta / k h \\
& \gamma_{s}=0,35+2,18 \beta / k h
\end{aligned}
$$

L'écart entre ces différentes relations empiriques a récemment été discuté par Sénéchal et al. (2005) lesquels ont mis en évidence l'influence du choix des fréquences de coupure spectrales sur le calcul de $\gamma_{\mathrm{s}}$. En zone de déferlement, la 


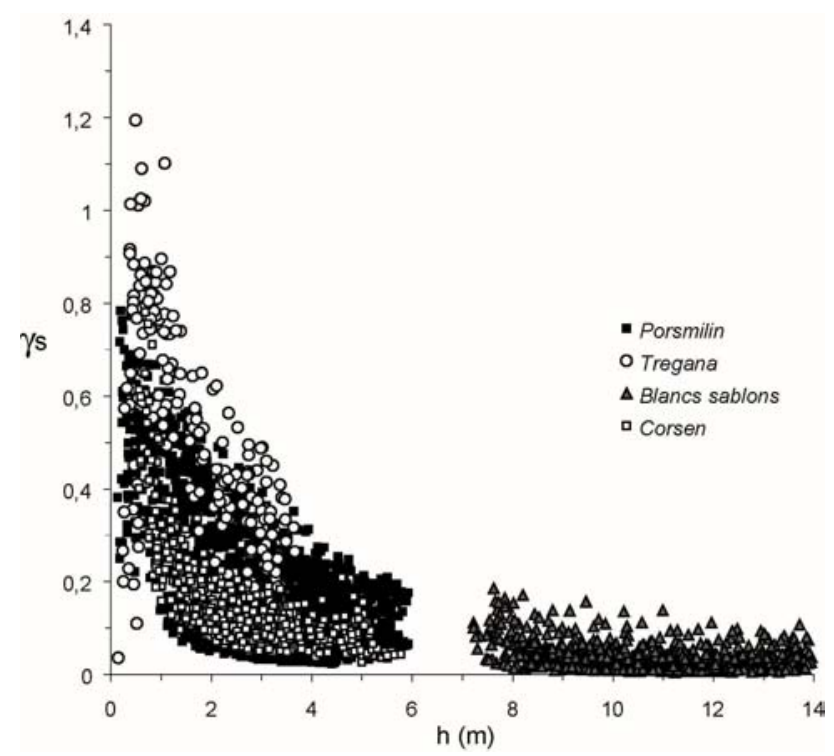

Figure 2. Hauteur relative des vagues $\gamma_{s}$ fonction de la hauteur d'eau locale $h$ sur les quatre plages.

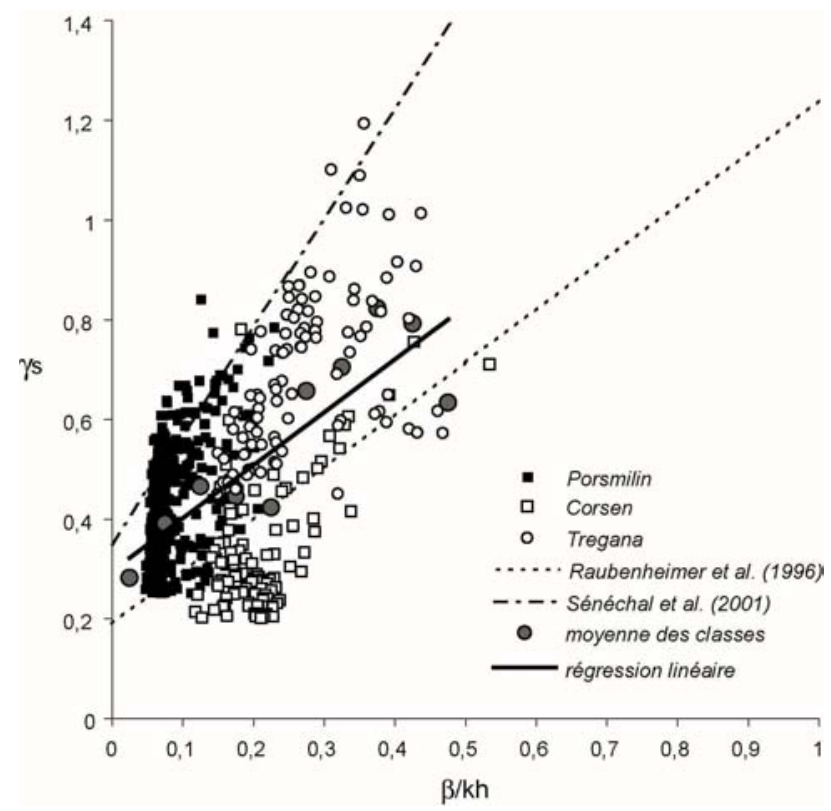

Figure 3. Hauteur relative des vagues $\gamma_{s}$ en zone de déferlement fonction de la pente locale normalisée $\beta / \mathrm{kh}$ pour les trois plages de Porsmilin, Corsen et Tregana. 
hauteur relative des vagues croît linéairement avec des pentes locales normalisées fortes c'est-à-dire dans des hauteurs d'eau faibles et/ou pour des pentes de plage fortes. Ceci implique que le taux de dissipation de l'énergie des vagues (par unité de distance) est minimal sur les plages dissipatives (aux Blancs Sablons, en bas d'estran à Porsmilin) et maximal sur les plages réflectives (Corsen, Tregana). Toutefois, la largeur de la zone de surf variant considérablement d'un type de plage à l'autre (de plusieurs centaines de mètres parfois à quelques mètres), il en résulte une dissipation de l'énergie des vagues quasiment totale sur les plages dissipatives, et à l'inverse, partielle sur les plages réflectives.

\subsection{Courants induits par les vagues}

La hauteur relative des vagues $\gamma_{\mathrm{s}}$ est employée pour décrire les variations spatiotemporelles de l'intensité des courants horizontaux au cours du cycle de marée et du déplacement des zones d'occurrence des processus hydrodynamiques. La figure 4 représente ainsi les courants moyens cross-shore $\mathrm{U}$ et longshore $\mathrm{V}$ fonction de la hauteur relative des vagues. Dans la zone intertidale, les données de vitesse des courants horizontaux s'organisent en trois groupes plus ou moins bien structurés selon les sites. Le premier groupe (noté 1) correspond à des courants quasi-nuls, inférieurs à $0,05 \mathrm{~m} / \mathrm{s}$, pour des faibles hauteurs relatives de vagues c'est-à-dire en zone de levée des vagues. Le second groupe (noté 2) est associé à des données acquises en zone de déferlement dans laquelle un courant cross-shore dirigé vers le large est observé. Ce courant devient significatif au-dessus d'une vitesse seuil de $0,05 \mathrm{~m} / \mathrm{s}$ (symbolisée par une ligne en pointillés sur la fig. 4) qui permet d'identifier la hauteur relative des vagues au point de déferlement $\left(\gamma_{\mathrm{s}, \mathrm{b}}\right)$. Elle constitue un critère de déferlement empirique et local qui varie de 0,2 à 0,45 selon les plages $\left(\gamma_{\mathrm{s}, \mathrm{b}}=0,2\right.$ à Corsen, 0,25 à Porsmilin, 0,45 à Tregana). En zone de déferlement, le courant de retour est dirigé vers le large car il compense d'une part, les flux de masse induits par les vagues et orientés vers la côte et d'autre part, les gradients de pression orientés vers le large (dus au set-up). Il atteint des vitesses moyennes de $0,25 \mathrm{~m} / \mathrm{s}$ (Corsen) à $0,45 \mathrm{~m} / \mathrm{s}$ (Porsmilin et Tregana) lors de conditions énergétiques $\left(\gamma_{\mathrm{s}}=0,4-\right.$ $0,8)$. Cette gamme de vitesse couvre à la fois les valeurs de $0,2-0,3 \mathrm{~m} / \mathrm{s}$ couramment reportées dans la littérature (Greenwood et Osborne, 1990; Masselink et Black, 1995 ; Anthony et al., 2004) mais aussi celles de 0,4 m/s plus rarement observées (Garcez Faria et al., 2000 ; Aagaard et al., 2005). En zone de déferlement, un courant longitudinal est également enregistré dont la vitesse moyenne peut atteindre $0,6 \mathrm{~m} / \mathrm{s}$ et dont la direction correspond à l'incidence des vagues. Enfin, le troisième groupe de données (noté 3 ) est associée aux hauteurs relatives de vagues les plus grandes enregistrées en zone de déferlement interne, à proximité de la zone de swash. On y observe une résultante du courant cross-shore pouvant être dirigée vers le large mais nettement diminuée, ou dirigée vers la plage (fig. 4). En effet, deux composantes de courant se superposent dans cette zone, l'une induite par les vagues déferlantes en surface et dirigée vers la plage, l'autre par le courant de retour dans la 

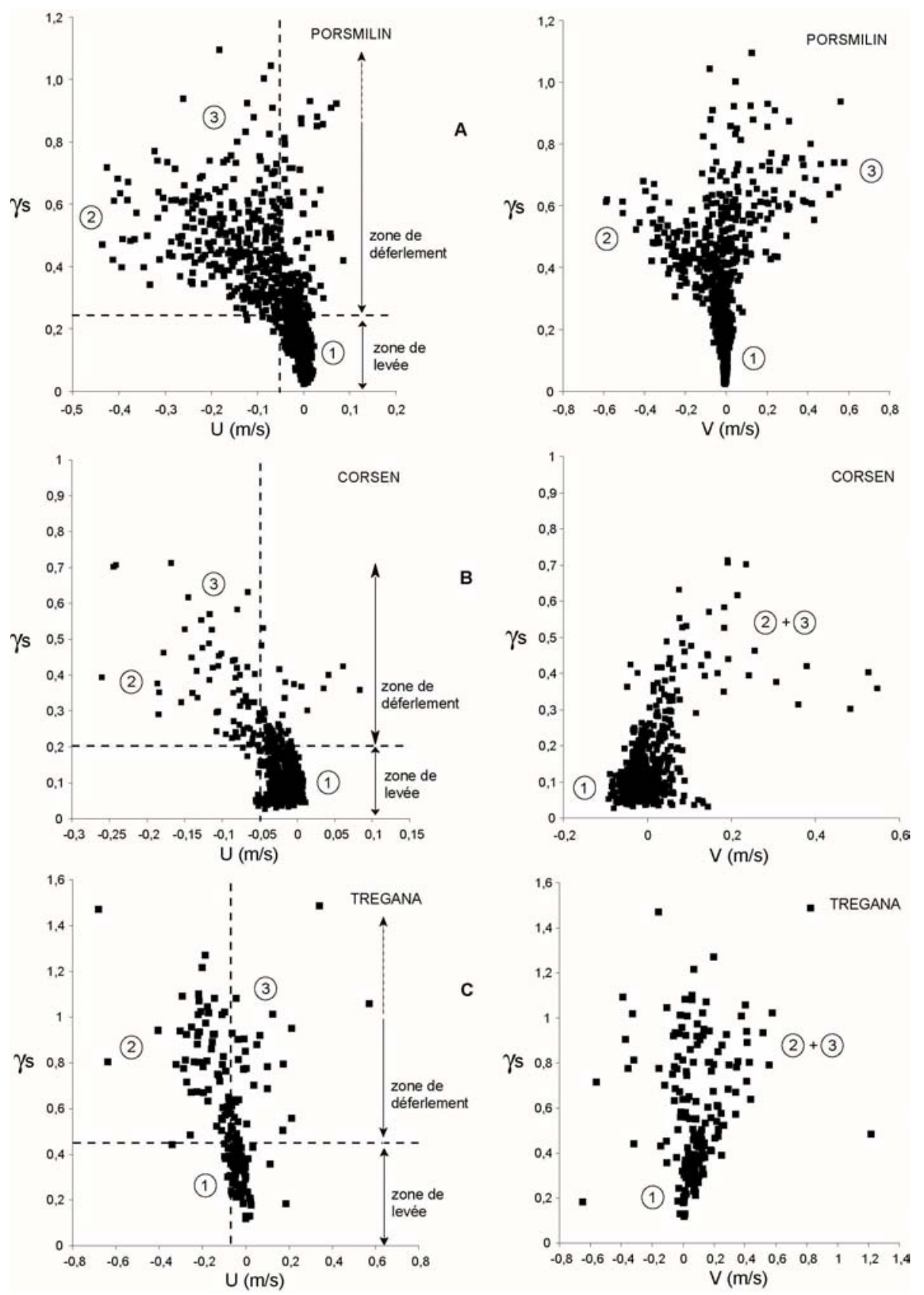

Figure 4. Courants cross-shore $U(U>0$ vers la plage, $<0$ vers le large) et longshore $V$ fonction de la hauteur relative des vagues $\gamma_{s}=H_{s} / h$ sur les plages de Porsmilin, Corsen et Tregana. 
partie inférieure de la colonne d'eau et dirigé vers le large. Lorsque la contribution des vagues déferlantes est supérieure à celle du courant de retour, on constate une «bascule» des courants, la composante cross-shore s'orientant vers la plage et la composante longitudinale dans le sens de propagation des vagues. La dissipation de l'énergie des vagues sur les plages, paramétrée par la hauteur relative, induit donc une spatialisation des processus hydrodynamiques et des courants qu'ils engendrent, schématisée par la figure 5. Ces résultats identifient trois régimes hydrodynamiques, (1) l'absence de courants induits par les vagues en zone de levée, (2) l'activation du courant de retour et d'un courant longitudinal en zone de surf externe, (3) la compétition entre le courant de retour et les déferlantes en zone de surf saturé.

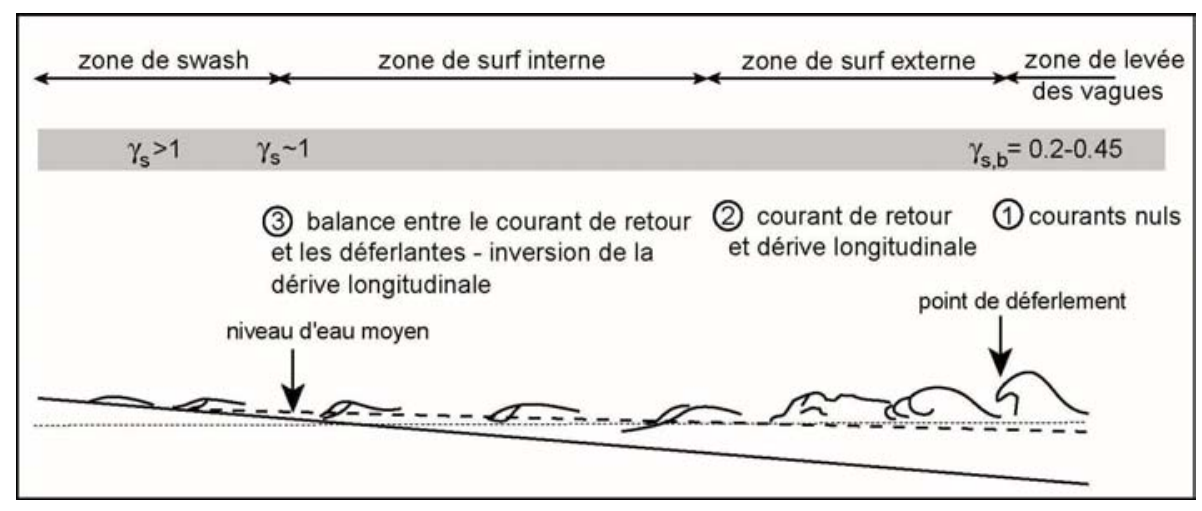

Figure 5. Evolution de la hauteur relative des vagues et type de courants induits lors de la transformation des vagues dans les petits fonds.

Si la structure en trois étapes est relativement évidente pour les données acquises à Porsmilin (fig. 4a), elle l'est beaucoup moins pour les données de Corsen et Tregana (fig. $4 \mathrm{~b}$ et c). Deux explications sont suggérées: à Corsen, la dérive longitudinale est exclusivement dirigée vers le nord $(\mathrm{V}>0)$ du fait de l'obliquité très forte des vagues de secteur sud pendant la totalité de la campagne de mesures ; à Tregana, on a observé un forçage bi-directionnel avec des vagues d'incidence SSO les premiers jours basculant SSE les derniers jours de la campagne de mesures. Ceci se traduit sur la fig. 4c par deux directions d'orientation du courant longitudinal qui semblent à première vue indifféremment corrélées à la hauteur relative des vagues.

Par une approche originale, la définition d'un critère de déferlement empirique et local a mis en évidence la contribution relative des différents processus hydrodynamiques (levée des vagues, courant de retour et vagues déferlantes) dans le transport sédimentaire en zone intertidale. Outre sa capacité à décrire les conditions d'énergie propices au déferlement des vagues et spécifiques à chaque plage, il pourra être exploité, dans le futur, dans des modèles empiriques de transport 
sédimentaire pour déterminer les conditions déferlantes de celles non déferlantes (Ruessink et al., 1999 ; Ruessink et Terwindt, 2000).

\section{Conclusion}

Les quatre jeux de données ont permis de décrire l'évolution spatio-temporelle des processus hydrodynamiques et du type de courants induits par les vagues au cours du cycle tidal sur les plages de la mer d'Iroise. En zone de déferlement, les principaux agents du transport sédimentaire sont le courant de retour dirigé vers le large, la dérive longitudinale et la composante de courant induite par les vagues déferlantes dirigée vers la plage. Pour cela, la hauteur relative des vagues $\gamma_{\mathrm{s}}$ a été utilisée comme indicateur de la dissipation de leur énergie lors de leur propagation depuis la zone de levée jusqu'en zone de déferlement interne. Du fait des nombreux processus affectant la propagation des vagues en zone littorale (effet de la hauteur d'eau, de la pente de la plage, des interactions entre triplets de fréquence), des lacunes persistent dans les modèles hydrodynamiques pour décrire avec réalisme la hauteur des vagues en zone de surf. Cette étude propose une nouvelle paramétrisation empirique qui, compilée aux observations existantes, permet d'affiner les formulations actuellement en vigueur. Par ailleurs, l'établissement d'un critère de déferlement $\left(\gamma_{\mathrm{s}, \mathrm{b}}\right)$ sur la base de l'activation du courant de retour, bien qu'empirique et local, a permis de départager les conditions d'énergie déferlantes de celles non déferlantes. Outre l'accord de ce critère aux valeurs rencontrées sur d'autres sites expérimentaux, sa prise en compte dans des modèles de transport sédimentaire permettra de caractériser dans l'espace et le temps l'occurrence relative des processus hydrodynamiques et de quantifier leur contribution au transport sédimentaire total par le biais des courants qu'ils induisent.

\section{Remerciements}

Les auteurs remercient G. Amis, G. Goasguen, A. Le Berre du CETMEF, F. Ardhuin et D. Corman du SHOM, P. Bretel de l'UMR EPOC Bordeaux 1, pour leur assistance sur le terrain. Les travaux de A. Dehouck ont été financés par une allocation doctorale de recherche du Ministère de l'Education Nationale et de la Recherche.

\section{Bibliographie}

Aagaard T., Kroon A., Andersen S., Moller Sorensen R., Quartel S., Vinther N., "Intertidal beach change during storm conditions; Egmond, The Netherlands", Marine Geology, vol. $218, n^{\circ} 1-4,2005$, p. $65-80$. 
Andersen O. H., Fredsoe J., Transport of suspended sediment along the coast, Report Progress report $\mathrm{n}^{\circ} 59$, Insitute of Hydrodynamics and Hydraulic Engineering, ISVA, Technical University of Denmark, 1983, 33-46 p.

Anthony E. J., Levoy F., Monfort O., "Morphodynamics of intertidal bars on a megatidal beach, Merlimont, Northern France”, Marine Geology, vol. 208, n¹, 2004, p. 73-100.

Dehouck A. Morphodynamique des plages sableuses de la mer d'Iroise (Finistère), Thèse de doctorat, Université de Bretagne Occidentale, 2006.

Dehouck A., Rihouey D., "Mid-term morphodynamics of the Iroise sea sandy beaches using linear statistical methods", Continental Shelf Research, submitted.

Dehouck A., Dupuis H., Amis G., Le Berre A., “An hydrodynamic field experiment in the Iroise sea, Porsmilin and Blancs Sablons study beaches". Proceedings of Coastal Dynamics 2005, Barcelona (Spain), 4-8 avril 2005, Sanchez-Arcilla A. (dir.), American Society of Civil Engineers, $15 \mathrm{p}$.

Garcez Faria A. F., Thornton E. B., Lippmann T. C., Stanton T. P. (2000)., "Undertow over a barred beach", Journal of Geophysical Research, vol. 105, n ${ }^{\circ}$ 7, p. 16999-17010.

Greenwood B., Osborne P. D., "Vertical and horizontal structure in cross-shore flows: An example of undertow and wave set-up on a barred beach", Coastal Engineering, vol. 14, $\mathrm{n}^{\circ} 6,1990$, p. 543-580.

Masselink G., "Simulating the effects of tides on beach morphodynamics", Journal of Coastal Research, vol. SI 15, 1993, p. 180-197.

Masselink G., Short A. D., "The effect of tide range on beach morphodynamics and morphology: a conceptual beach model”, Journal of Coastal Research, vol. 9, n³, 1993, p. $785-800$.

Masselink G., Black K. P., "Magnitude and cross-shore distribution of bed return flow measured on natural beaches", Coastal Engineering, vol. 25, n³-4, 1995, p. 165-190.

Masselink G., Turner I. L., The effects of tides on beach morphodynamics, Handbook of beach and shoreface morphodynamics, Short, A. D. (dir.), Wiley, 1999, p. 204-229.

Osborne P. D., Greenwood B., "Frequency dependent cross-shore suspended sediment transport: 2. A barred shoreface", Marine Geology, vol. 106, 1992, p. 25-51.

Raubenheimer B., Guza R. T., Elgar S., "Wave transformation across the inner surf zone". Journal of Geophysical Research, vol. 101, n ${ }^{\circ} \mathrm{C} 10,1996$, p. 25589-25597.

Ruessink B. G., Houwman K. T., Hoekstra P., "The systematic contribution of transporting mechanisms to the cross-shore sediment transport in water depths of 3 to $9 \mathrm{~m}$ ", Marine Geology, vol. 152, n²4, 1998, p.295-324.

Ruessink B. G., Houwman K. T., Hoekstra P., "Medium-term frequency distributions of cross-shore suspended sediment transport rates in water depths of 3 to 9 m", Coastal Engineering, vol. 38, n²1, 1999, p. 25-46.

Ruessink B. G., Terwindt J. H. J., "The behaviour of nearshore bars on the time scale of years: a conceptual model”, Marine Geology, vol. 163, n¹-4, 2000, p. 289-302. 
12 REGC - /2007. Génie côtier

Sénéchal N., Dupuis H., Bonneton P., Howa H., Pedreros R., "Observation of irregular wave transformation in the surf zone over a gently sloping sandy beach on the French Atlantic coastline", Oceanologica Acta, vol. 24, nº, 2001, p. 545-556.

Sénéchal N., Rey V., Bonneton P., Dupuis H., "On the difficulty of correctly determine the characteristic wave period in the surf zone", Proceedings of Coastal dynamics 2005, Barcelona (Spain), 4-8 avril 2005, Sanchez-Arcilla A. (dir.), American Society of Civil Engineers, $12 \mathrm{p}$.

Thornton E. B., Guza R. T., "Energy saturation and phase speeds measured on a natural beach", Journal of Geophysical Research, vol. 87, n ${ }^{\circ} 12$, 1982, p. 9499-9508.

Thornton E. B., Guza R. T., "Transformation of wave height distribution", Journal of Geophysical Research, vol. 88, 1983, p. 5925-5938. 